Please do not remove this page

RMIT

UNIVERSITY

\title{
Modelling and design of extremely low frequency uniform magnetic field exposure apparatus for in vivo bioelectromagnetic studies
}

Cvetkovic, Dean; Cosic, Irena

https://researchrepository.rmit.edu.au/esploro/outputs/9921863802501341/filesAndLinks?institution=61RMIT_INST\&index=null

Cvetkovic, D., \& Cosic, I. (2007). Modelling and design of extremely low frequency uniform magnetic field exposure apparatus for in vivo bioelectromagnetic studies. Proceedings of the 29th Annual International Conference of the IEEE EMBS, Lyon, France, 1675-1678. https://doi.org/10.1109/IEMBS.2007.4352630

Published Version: https://doi.org/10.1109/IEMBS.2007.4352630

Repository homepage: https://researchrepository.rmit.edu.au

(c) 2007 IEEE. Personal use of this material is permitted. However, permission to reprint/republish this material for advertising or promotional purposes or for creating new collective works for resale or redistribution to servers or lists, or to reuse any copyrighted component of this work in other works must be obtained from the IEEE.

Downloaded On 2023/04/26 22:39:48 +1000 


\title{
Aunust $232.26,2007$. \\ Modelling and Design of Extremely Low Frequency Uniform Magnetic Field Exposure Apparatus for In Vivo Bioelectromagnetic Studies
}

\author{
D. Cvetkovic and I. Cosic, Senior Member
}

\begin{abstract}
Helmholtz coils are regularly utilised for various extremely low frequency (ELF) bioelectromagnetic experiments. The evaluation was conducted for the Helmholtz coil magnetic field frequency and uniformity, characterised by frequency-domain and geometric ELF magnetic exposure characteristics. An established approach which consisted of the mathematical calculations of the geometric parameters, computational modeling, and experimental development measurements of the Helmholtz coil's magnetic field frequency and uniformity, improved the quality of magnetic field uniformity and minimised the magnetic field intensity losses.
\end{abstract}

\section{INTRODUCTION}

$\mathrm{H}$ elmholtz coils are commonly used for exposing biological systems such as cells and human subjects under the assumption that the field in the region occupied by the biological systems is relatively uniform [1-3]. However, a common mistake in bioelectromagnetic experiments is an assumption that Helmholtz coils provide uniform magnetic fields and that the magnitude of that field is constant throughout the entire ELF $(0-300 \mathrm{~Hz})$ spectrum. The magnetic field uniformity appears to be realistic only for a limited volume at the center whereas the level of that same field is limited due to magnetic field frequency influenced by the mutual inductance and impedance matching.

A great precautions need to be taken in order to characterise a particular 'ELF magnetic field exposure'. The two major EMF exposure categories are the frequency of field oscillations (frequency-domain characteristics) and spatial homogeneity (geometric characteristics) [4]. The purpose of this research is to evaluate the ELF limitations of the Helmholtz coil magnetic field frequency and uniformity which are characterised by those two major EMF exposure categories. This evaluation will be undertaking by conducting a computational modeling and experimental measurements from the developed EMF exposure system.

Manuscript received April 2, 2007.

D. Cvetkovic (corresponding author) is with Australian Centre for Radiofrequency Bioeffects Research (ACRBR) and RMIT University, School of Electrical and Computer Engineering, GPO Box 2476V, Melbourne, VIC 3001, Australia, tel: +613 9925 9641, fax: +613 9925 2007, e-mail: dean.cvetkovic@rmit.edu.au.

I. Cosic is with Australian Centre for Radiofrequency Bioeffects Research (ACRBR) and RMIT University, School of Electrical and Computer Engineering, GPO Box 2476V, Melbourne, VIC 3001, Australia, tel: +613 9925 1971, fax: +613 9925 2007, e-mail: irena.cosic@ rmit.edu.au.

\section{MATERIALS AND METHODS}

\section{A. Theory of Helmholtz Coil Uniform Magnetic Field}

The time-harmonic (AC) equation with the magnetic vector potential $A$ is expressed as:

$j \omega \sigma A+\nabla \times\left(\mu^{-1} \nabla \times A\right)=J^{e}$

where $\Phi$ is the angular frequency, $\sigma$ is the conductivity, $\mu$ is the permeability and $J^{e}$ is the external applied current density.

In the case of conductive media, the current density is described using Maxwell-Ampere's law for quasi-static systems as [5]:

$J=\sigma(E+v \times B)+J^{e}$

where $B$ is the magnetic flux density (Tesla), $E$ is electric field intensity and $v$ is the velocity.

The flux density $B$ at each point within the coil is obtained by solving the Biot and Savart equations:

$B=\nabla \times A=\nabla \times \frac{\mu_{0} I}{4 \pi} \int \frac{d s}{R}$

where $\nabla$ is the gradient, $\mu_{0}$ is permeability of free space $4 \pi \times 10^{-7} \mathrm{H} / \mathrm{m}, I$ is the coil current (A), $R$ is the distance from a point of integration on the circuit to $P$ point of observation (m) and $d s$ is an infinitesimal segment of the current loop.

In a simplified formulation, when including the number of turns for each coil, then the magnetic field intensity at any point on the axis of the coils is [6]:

$$
B(z)=\frac{1}{2} \mu_{0} N I R^{2}\left\{\left[R^{2}+\left(z+\frac{R}{2}\right)^{2}\right]^{-3 / 2}+\left[R^{2}+\left(z-\frac{R}{2}\right)^{2}\right]^{-3 / 2}\right\}
$$

(4)

At the centre of Helmholtz coils, the magnetic field intensity is [7]:

$$
B(0)=\frac{16}{5 \sqrt{5}} \frac{1}{2} \frac{\mu_{0} N I}{R}=1.43 \frac{\mu_{0} N I}{D}
$$

The resistance of the coil is given by

$R=\rho l / s=4 \rho l / \pi d^{2}$

which gives

$$
l=R \pi d^{2} / 4 \rho=N \pi D
$$


where $\rho$ is the resistivity of the material (copper $1.7 \times 10^{-8}(\Omega m), l$ is the length of wire, $s$ and $d$ are the cross section and the diameter of the wire respectively and $D$ is the coil diameter [7].

One of the basic limitations in available frequency is the rise time of the field in the coil, due to its inductance $L$ [7].

$$
L=\mu_{0} D N^{2}\left[\ln (8 D / d)-\frac{7}{4}\right]
$$

In the Helmholtz configuration, the mutual inductance $M$ between the coils is also taken into consideration. Therefore, the maximum magnetic field frequency is [7]:

$$
f_{\max }=\frac{32 \rho^{2} D}{4.4 \mu_{0} R d^{4}[\ln (8 D / d)-1.36]}
$$

There are several limitations, which need to be taken into an account for experimental bioelectromagnetic work, such as a relation between the intensity of the magnetic field and the frequency, which needed to be considered in this investigation. In the Helmholtz configuration, the performance depends upon the required magnetic field level, frequency and source power. First, the coils and source must be matched to maximise the magnetic field. This is accomplished by setting the number of turns to equate the source impedance with the coil reactance. Magnetic field intensity loss is one of the basic limitations where for the applied frequency, the rise time of the magnetic field in the coil is influenced by its inductance $L$ and mutual inductance $M$. The inductance is determined by the coil diameter $D$, number of wire turns in a coil $N$ and the wire diameter $d$ parameters. The maximum magnetic field frequency, $f_{\max }$ eq. (9) can not overcome the resulting rise time between $10 \%$ and $90 \%$ of the maximum field intensity, given by $\theta r=2.2 \mathrm{Lr} / \mathrm{R}$, where $R$ is radius of the coil and $L r$ is the resulting inductance, $L r=L+M$ [7]. A magnetic field intensity loss occurs if the applied frequency is increased more than the maximum limited frequency, fmax. Therefore, it is very important to determine the geometric parameters of the coil for the applied frequency.

\section{B. Computational Modelling and Simulation}

Computational modelling and simulation of ELF uniform magnetic field exposure system prior to the actual practical design and development process is very important in terms of design flexibility and efficiency. 3-D Finite Element Method (FEM) modeling and simulation software Femlab 3.1 (Comsol Inc.) [8] has been utilised to computationally model and simulate Helmholtz coil system for the most effective magnetic field uniformity. Our model has utilised 3D FEM quasi-static time-harmonic application mode. Helmholtz coils modelled and designed for this investigation were of circular shape, with radius of $0.65 \mathrm{~m}, 250$ turns, current of approximately $140 \mathrm{~mA}$ and resultant current density of $280 \cdot 10^{3}\left(\mathrm{~A} / \mathrm{m}^{2}\right)$. The total applied voltage and coil impedance was $12 \mathrm{~V}$ and $70 \Omega$ at applied frequency of $8.33 \mathrm{~Hz}$. Mathematical calculations of coil physical dimensions, coil turns and current densities were performed to model two symmetrical geometrical coils generate the required uniform magnetic field intensity [4]. In computing more accurate results a refined meshing within the volume specified for magnetic field uniformity was performed. Copper material was assigned for conductive coils with conductivity of $\sigma=5.998 \times 10^{7} \mathrm{~S} / \mathrm{m}$. The currents specified for both models were in phase for the symmetrical coils.

\section{Design of Helmholtz Coil Uniform Magnetic Field Exposure Apparatus}

The exposure system that was used in this particular investigation was a standard double Helmholtz coil, developed on a $1 / 2$ inch thick plywood circular frame of radius of $64.3 \mathrm{~cm}$, average radii of $65 \mathrm{~cm}$ [1-3], as shown in Fig. 1. The copper wires of $0.8 \mathrm{~mm}$ in diameter (20 AWG) and cross-sectional area of $0.5156 \mathrm{~mm}^{2}$ were professionally machine-wounded and layered from left to right hand side in rectangular form, with 250 turns each, inside a bobbin made of high-impact PVC-U mini trunks. An ELF (0-50Hz) signal generator was designed and developed using EXAR XR-2206 monolithic IC, capable of producing high quality sine, square, triangle, ramp and pulse waveforms of high stability and accuracy, with typical low-sine wave distortion of $0.5 \%$. In order to design a sine-wave generator with a minimum harmonic distortion and stable and accurate frequency, a selection of passive components was required to control the required variable output, signal symmetry, frequency and dc offset. The magnetic flux density output of $20 \mu \mathrm{T}$ (rms) was generated by $12 \mathrm{~V}$ source from the developed audio amplifier with the fixed gain of 10 .

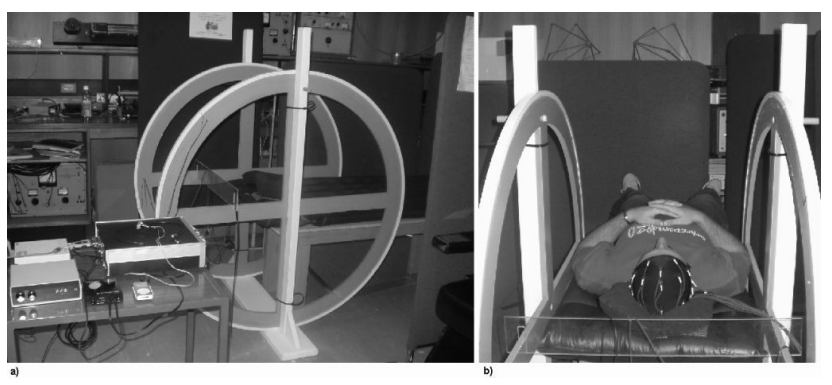

Figure 1. Helmholtz coil ELF uniform magnetic field exposure apparatus for In Vivo bioelectromagnetic studies [2].

Primarily, the Helmholtz coil was constructed for a purpose of exposing human subjects at the frequency of $8.33 \mathrm{~Hz}$ and magnetic field of $20 \mu \mathrm{T}$ (rms), as shown in Fig. 1. No mechanical vibrations were generated by magnetic field exposure coils throughout the testing stage. The linearly polarized filed was perpendicular to the Earth's North-South magnetic field. The magnetic flux density was verified by direct measurement using "Wandel and Goltermann" EFA-200 EMF Analyser and external B-field probe with diameter of $3 \mathrm{~cm}$ and measurement accuracy of $6 \%$. In order to maximise the accuracy and the efficiency of the magnetic field measurements, an apparatus for mapping the magnetic flux density was developed. This mapping apparatus consisted of a plastic arm mounted on a two-dimensional meter. The magnetic flux density probe was mounted on the 
arm which allowed movements along the z-axis direction and the arm mounted on the two-dimensional meter allowed for movements along the $x$-y-axes direction. The design of the new measurement apparatus excluded the use of any conductive materials that could possibly alter the magnetic field distribution within the coils. There were $65 \times 30 \times 65(5 \mathrm{~cm}$ interval) probe positions mapped for the complete magnetic field uniformity measurements. In order to efficiently determine the uniformity of magnetic field generated by the Helmholtz coils, the coordinates selected were the along axes passing through the origin, such as points along the $\mathrm{x}$-axis $(\mathrm{z}, \mathrm{y}=0), \mathrm{y}$-axis $(\mathrm{x}, \mathrm{z}=0)$ and $\mathrm{z}$-axis at $(\mathrm{x}, \mathrm{y}=0)$. The measurements were systematically saved in the Field Analyser and the data was imported via a serial port to Data Logger software as an ASCII file. The data was then imported into Matlab (Mathworks Inc.) and tabulated to graph the magnetic field measurements correctly against its displacements.

\section{RESULTS}

The computational magnetic field uniformity post-processing was conducted by plotting a radial magnetic flux density distribution for an axial $B(x, y, z)$ positions within the coils. Fig. 2 a) shows the computed maximum magnetic flux density of $42.5 \mu \mathrm{T}$ (peak) or $30.1 \mu \mathrm{T}$ (rms). From the plot shown in Fig. 2a), it was evident that the interior boundary condition specified by a spherical volume was effective in smoothing out the edges of curves within $\pm 55 \mathrm{~cm}$ region. Uniformity levels from the inner to the outer region were $0.01 \%, 0.1 \%$ and $1 \%$ with respect to the centre value. The inner level of $0.01 \%$ has shown the magnetic field uniformity of $26.43 \mathrm{~cm}$ (x-axis), $16.03 \mathrm{~cm}$ (y-axis) and $30.06 \mathrm{~cm}$ (z-axis) or within volume of $12,735 \mathrm{~cm}^{3}$ (Table I). At outer level of $1 \%$, the uniformity was $56.52 \mathrm{~cm}$ (x-axis), $42.48 \mathrm{~cm}$ (y-axis) and $45.69 \mathrm{~cm}$ (z-axis) within volume of $109,700 \mathrm{~cm}^{3}$.

According to magnetic flux density measurements acquired, the uniformity of the inner level, $0.01 \%$, was $15 \mathrm{~cm}$ ( $\mathrm{x}$ and $\mathrm{y}$ axes) and the outer level $1 \%$ was $40 \mathrm{~cm}$ (x-axis) and $50 \mathrm{~cm}$ (y-axis), as shown in Fig. 2b) and Table 1. The uniformity at the z-axis was irregular and was therefore excluded from further analysis. The measured ambient or geomagnetic field inside the RF shielded room was approximately $200 \mathrm{nT}$ at the ELF range $6-11 \mathrm{~Hz}$.

In terms of frequency of field oscillations, mathematical design calculations, practical development and measurements of magnetic field from the Helmholtz coil have assisted in matching the source impedance with the coil reactance and taking into account the mutual inductance between the two coils. Magnetic flux density was measured at the origin $(x=0$, $\mathrm{y}=0, \mathrm{z}=0$ ) in the frequency range $0-50 \mathrm{~Hz}$. The computational results of the magnetic field uniformity were recorded at $42.5 \mu \mathrm{T}$ (peak) at $8.33 \mathrm{~Hz}$, whereas, the maximum magnetic field frequency was measured to be $42.2 \mu \mathrm{T}$ (rms) at $8.5 \mathrm{~Hz}$.
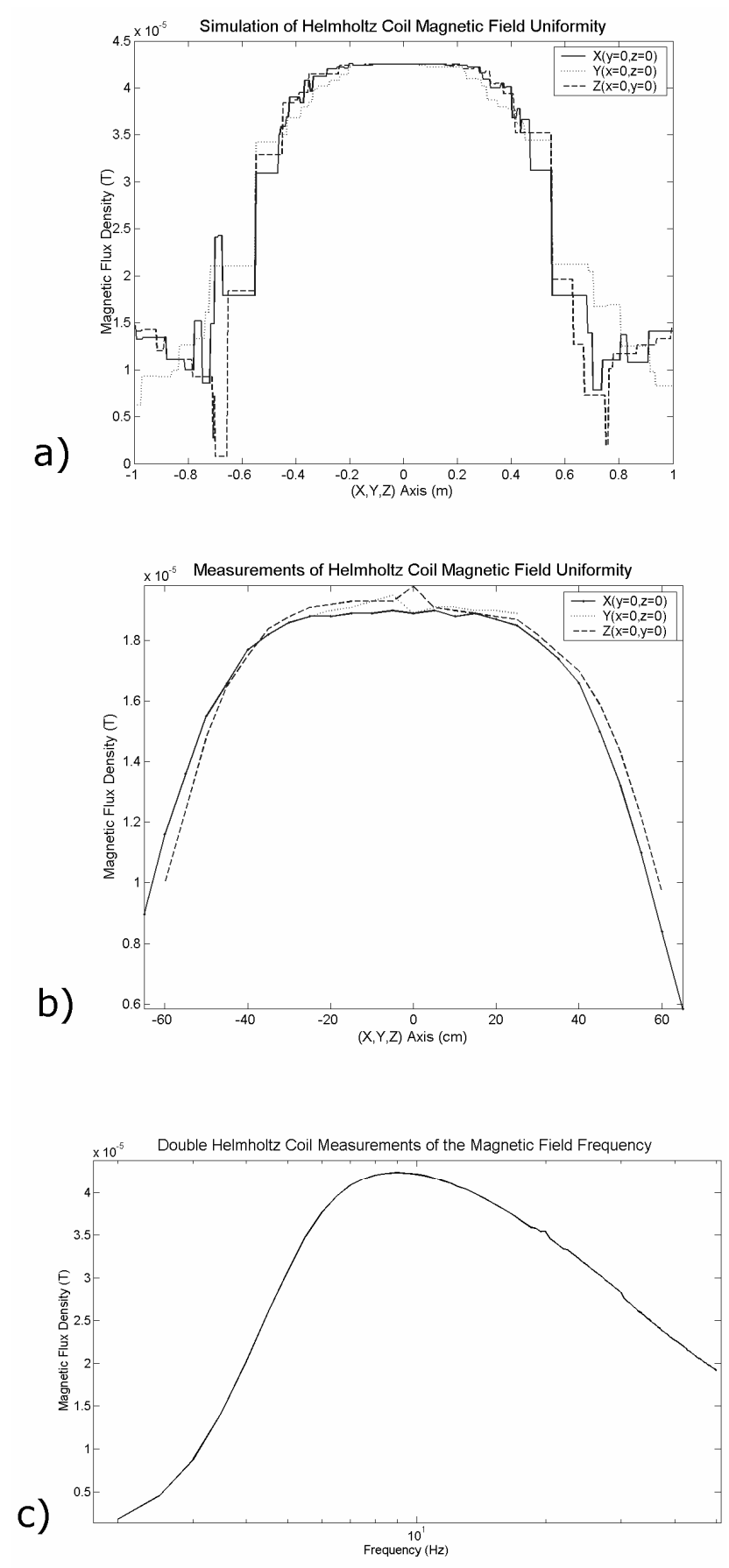

Figure 2 a) Radial magnetic flux density distribution for an axial $(x, y, z)$ position between the coils of the computational model and b) constructed experimental Helmholtz coils. c) Magnetic flux density, generated by applied frequency of $8 \mathrm{~Hz}$ was measured from the constructed Helmholtz coils which showed that the magnetic flux density along radial $\mathrm{x}$-axis (represented on the $\mathrm{y}$-axis of the plot) was maximum at $8.5 \mathrm{~Hz}$. This result indicated the precise impedance matching between the signal generator and the coils. The $\mathrm{x}$-axis of the plot is represented by the frequency $(\mathrm{Hz})$ on a logarithmic scale.

As the frequency was increased to $50 \mathrm{~Hz}$, the actual magnetic flux density decreased to $20 \mu \mathrm{T}$, as shown in Fig. $2 \mathrm{c})$. As required, the amplitude from the signal generator was adjusted to match the maximum magnetic flux density of 
$20 \mu \mathrm{T}$ (rms) to $8 \mathrm{~Hz}$. Our Helmholtz coils were designed and constructed to ensure the matching of source impedance with the coil reactance, exact series inductance and mutual inductance between the coil pair.

TABLE I

COMPUTATIONAL AND EXPERIMENTAL RESULTS OF MAGNETIC FIELD UNIFORMITY AT $0.01 \%, 0.1 \%$ AMD $1 \%$ LEVELS, CALCULATED AT X, Y AND Z AXES.

\begin{tabular}{ccccccc}
\hline \multirow{2}{*}{$\begin{array}{l}\text { Uniformity } \\
\text { level }\end{array}$} & \multicolumn{3}{c}{ Model } & \multicolumn{3}{c}{ Measurements } \\
\cline { 2 - 7 } & X-axis & Y-axis & Z-axis & X-axis & Y-axis & $\begin{array}{l}\text { Z- } \\
\text { axis }\end{array}$ \\
\hline $0.01 \%$ & 26.43 & 16.03 & 30.06 & 15.00 & 15.00 & \\
$0.1 \%$ & 42.09 & 27.66 & 45.29 & 15.00 & 40.00 & NA \\
$1 \%$ & 56.52 & 42.48 & 45.69 & 40.00 & 50.00 & \\
\hline
\end{tabular}

For the spatial homogeneity of magnetic field, the modeling and simulations results were compared and validated with the experimentally measured magnetic field results from the developed Helmholtz coils. The error difference between the computational and measured magnetic field uniformity results at inner level of $0.01 \%$ was $43 \%$ (x-axis), $6 \%$ (y-axis); at level $0.1 \%$ was $64 \%$ (x-axis), $45 \%$ (y-axis); and at level $1 \%$ was $29 \%$ (x-axis) and $18 \%$ (y-axis). The smallest error difference was found at the $0.01 \%$ level along $\mathrm{x}$-axis. Considering that the magnetic flux density was measured at every $5 \mathrm{~cm}$, it caused difficulties in properly assessing and measuring the magnetic field uniformity. However, the FEM modeling method was better utilised in terms of accuracy and time consumption.

\section{DISCUSSION}

In this paper we have identified the two major factors that could improve future magnetic field exposure system designs for In Vivo and possibly applied for In Vitro studies. The two EMF exposure factors are the frequency of field oscillations (frequency-domain characteristics) and spatial homogeneity (geometric characteristics) previously reported by Valberg [4]. We have addressed characteristic issues and designed an approach which included the mathematical calculations of the geometric parameters, FEM modeling, development and experimental measurements of the Helmholtz coil's magnetic field uniformity. This approach was established in order to improve the quality of the magnetic field uniformity and minimisation of the magnetic field intensity losses. The improvement of the magnetic field uniformity was primarily done by refining the mathematical calculation and modeling solution of Helmholtz coil parameters, whereas the minimisation of the magnetic field losses was addressed practically by matching the source impedance with the coil reactance and taking into account the inductance and mutual inductance between the two coils [9], to maximise the magnetic field intensity at the applied frequency of $8.33 \mathrm{~Hz}$.

\section{CONCLUSION}

For the ELF bioelectromagnetic experiments, several theoretical, computational, and practical characteristics have been evaluated and developed in order to improve the quality of magnetic field exposure systems.

\section{ACKNOWLEDGMENT}

The authors gratefully acknowledge the NHMRC for their support of the ACRBR which has assisted this research work.

\section{REFERENCES}

[1] D. Cvetkovic, E. Jovanov, I. Cosic, "Alterations in Human EEG Activity Caused by Extremely Low Frequency Electromagnetic Fields, in Proc. $28^{\text {th }}$ IEEE EMBS Annual International Conference, New York City, USA, Sep 2006, pp. 3206-3209.

[2] D. Cvetkovic, "Electromagnetic and Audio-Visual Stimulation of the Human Brain at Extremely Low Frequencies" Ph.D. dissertation, School of Electrical and Computer Engineering, RMIT Univ., Melbourne, Australia, 2005.

[3] G.B. Bell, A.A. Marino, "Exposure system for production of uniform magnetic fields", Journal of Bioelectricity, vol. 8, no. 2, pp.147-158, 1989.

[4] P.A. Valberg, "Designing EMF experiments: What is required to characterize Exposure?", Bioelectromagnetics, vol. 16, pp. 396-401, 1995.

[5] Z. Haznadar, Z. Stih, Electromagnetic fields, waves and numerical methods, Amsterdam, NL, IOS Press, 2000.

[6] J. Wang, S. She, S. Zhang, "An improved Helmholtz coil and analysis of its magnetic field homogeneity", The Review of Scientific Instruments, vol. 73, no. 5, pp. 2175-2179, 2002.

[7] R. de Seze, A. Lahitte, J.M. Moreau, B. Veyret, "Generation of extremely-low frequency magnetic fields with standard available commercial equipment: implications for experimental bioelectromagnetics work", Bioelectrochemistry and Bioenergetics, vol. 35, pp. 127-131, 1994.

[8] Comsol, Femlab 3., Electromagnetics Module, Stockholm, Sweden: Comsol AB. pp. 43-50, 2004.

[9] L. Raganella, M. Guelfi, G. D'Inzeo, “Triaxial exposure system providing static and low frequency magnetic fields for in vivo and in vitro biological studies". Bioelectrochemistry and Bioenergetics, vol. 35, pp. 121-126, 1994. 